\title{
Ionospheric precursors to scintillation activity
}

\author{
Paul S.J. Spencer
}

European Commission, Joint Research Center (JRC), IPSC, I-21027 Ispra (Varese), Italy

\author{
Article history \\ Received July 29, 2013; accepted February 19, 2014. \\ Subject classification: \\ Scintillations, Forecasts, Dynamics, Inverse methods, Algorithms and implementation.
}

\begin{abstract}
Ionospheric scintillation is the rapid fluctuation of both phase and amplitude of trans-ionospheric radio waves due to small scale electron density irregularities in the ionosphere. Prediction of the occurrence of scintillation at $L$ band frequencies is needed to mitigate the disruption of space-based communication and navigation systems. The purpose of this paper is to present a method of using tomographic inversions of the ionospheric electron density obtained from ground-based GPS data to infer the location and strength of the post-sunset plasma drift vortex. This vortex is related to the pre-reversal enhancement in the eastwards electric field which has been correlated to the subsequent occurrence of scintillation.
\end{abstract}

\section{Introduction}

This paper presents preliminary work concerned with the prediction of scintillation observed on L-Band frequencies in the equatorial ionosphere. Equatorial scintillation is generally observed post-sunset and at the equinoxes. The post-sunset ionosphere is characterised by the formation of a drift vortex in the plasma at a local time of approximately 20LT and at a height of typically $225 \mathrm{~km}$ [Rodrigues et al. 2012]. The vortex occurs at the transition between the day-side $\mathrm{E}$ region and night-side F region dynamos. During the daytime, electric fields are eastwards and the plasma drifts westwards and upwards. This situation reverses during the night-time with westwards electric fields and eastwards/downwards plasma drifts. To the east of the vortex strong vertical drifts are observed which are associated with a pre-reversal enhancement in the eastwards electric field (PRE). This electric field drives the plasma upwards where the reduction in collision frequency on the bottom side ionosphere reduces the time scale for the formation of irregularities [Kudeki et al. 2007].

Anderson et al. [2004] first reported the possibility of forecasting equatorial scintillation at VHF/UHF frequencies based upon the strength of the pre-reversal enhancement. The FIRST model, Redmond et al. [2010], uses ionosonde measurements to obtain an es- timate of the rise in the post-sunset peak height of the ionospheric F2 layer. This measurement is then treated as a proxy for the pre-reversal enhancement in the equatorial electric field. An enhanced post sunset electric field is known to be related to the onset of plasma irregularities based upon the generalized Rayleigh-Taylor instability mechanism, Fejer and Kelley [1980].

The purpose of this paper is to present a method whereby tomographic inversions of the electron density using ground-based GPS observations may be used to provide an alternative data source to achieve similar results to those reported by Anderson. The MIDAS package, Mitchell and Spencer [2003], provides an estimate of the four-dimensional electron density in the ionosphere using ground-based GPS receivers. It is shown that the solution to the continuity equation applied to MIDAS estimates of vertical total electron content (TEC) provides signatures of the post-sunset drift vortex.

In order to test the method the results obtained are related to the occurrence of scintillation events observed by the COSMIC satellite constellation. COSMIC consists of six low Earth orbit satellites, each of which deploys a dual frequency GPS receiver primarily for use in radio occultation experiments. The data set are routinely processed to provide $\mathrm{S} 4$ estimates along each occultation path [e.g. Straus and Bishop 2009, Uma et al. 2009]. The advantage of using the COSMIC S4 scintillation estimates is that, with typically 300 occultation passes per day, global data coverage is available.

\section{Theory}

GPS inversions from the MIDAS package provide an estimate of the global ionospheric electron density to a resolution of approximately 5 degrees latitude and longitude and 20 minutes in time. Although the inversions provide an estimate of the vertical electron density profile, the method is limited in its vertical resolution 
due to geometrical factors. As such, isolating the vertical plasma drifts using MIDAS would be difficult at the required spatial and temporal resolutions. Tomographic methods using ground-based GPS do however provide accurate estimates of the integrated vertical total electron content. Consequently, the aim of this project was to derive a method that could relate signatures observed in the horizontal distribution of TEC to the post sunset drift vortex.

In order to infer the existence of the drift vortex using TEC, the zonal drift in the plasma was considered. An eastwards acceleration of the plasma above the vortex would be expected to lead to a zonal divergence in plasma velocity. Since most of the F-region plasma is located above the drift vortex, at typically $225 \mathrm{~km}$, this should also result in a zonal divergence in the vertically integrated TEC. By using TEC, this approach ignores the vertical redistribution of plasma that arises due to zonal electric fields and vertical drifts.

In order to obtain an estimate of the horizontal velocity of the vertically integrated TEC, the solution to the continuity equation was obtained from the MIDAS reconstructions. The continuity equation can be written

$$
\frac{\partial N}{\partial t}+\nabla \cdot(N \mathbf{v})=P-L
$$

Where $N$ is the known vertical total electron content, $\mathbf{v}$ the horizontal velocity, $t$ is time and the production and loss are given by $P$ and $L$ respectively. To remove the production and loss terms from Equation (1) the MIDAS reconstructions were computed in a solar magnetic coordinate system. Since the method presented is primarily concerned with the post sunset period, production in this region will be negligible. Furthermore, in the post-sunset F2 region, the increasing time constant for recombination and decreasing time constant for diffusion result in the transport of ionisation playing an increasingly important role, Bittencourt et al. [2007]. As such, the errors arising from ignoring the loss term at fixed solar magnetic coordinates should be small in relation to the other terms.

The solution of the continuity equation is nontrivial since with two velocity components and a single observation per pixel there are twice as many unknowns as knowns. To obtain stable solutions the problem was reformulated by defining the velocity, $\mathbf{v}$, as the gradient of a scalar potential field, $\phi$. In this case the continuity equation becomes

$$
\frac{\partial N}{\partial t}+N \nabla^{2} \phi+\nabla N \cdot \nabla \phi=0
$$

A limitation of this approach is that the resulting solution for the velocity is no longer general since it is constrained to be irrotational. However, for the current work this assumption was considered acceptable since horizontal plasma drifts in the equatorial F-region are predominantly zonal. Equation (2) was then solved for $\phi$ using a finite difference approach to the derivatives. Expanding the terms in Equation (1) gives

$N\left(\frac{\partial^{2} \phi}{\partial x^{2}}+\frac{\partial^{2} \phi}{\partial y^{2}}\right)+\frac{\partial N}{\partial x} \frac{\partial \phi}{\partial x}+\frac{\partial N}{\partial y} \frac{\partial \phi}{\partial y}=-\frac{\partial N}{\partial t}$

Where the first and second derivatives in the two horizontal spatial dimensions $x$ and $y$ are formulated using finite differences over three adjacent grid pixels. The Equation (3) can be then written in matrix form as

$$
\mathrm{H} \varphi=\mathrm{b}
$$

To ensure unique solutions the problem is reformulated as a set of regularised normal equations

$$
\left(\mathbf{H}^{T} \mathbf{H}+\lambda \mathbf{R}^{T} \mathbf{R}\right) \boldsymbol{\varphi}=\mathbf{H}^{T} \mathbf{b}
$$

Where $\mathbf{R}$ is a regularisation matrix and $\lambda$ is a constant. The matrix $\mathbf{R}$ is included to provide smoothing in the two spatial dimensions by minimizing the departure of the solution from a linear function using the kernel $[1,-2,1]$. The constant $\lambda$ is chosen so that the trace of the normal equations corresponding to the observation matrix $\mathbf{H}$ and regularisation matrix $\mathbf{R}$ have equal weight.

\section{Data}

MIDAS reconstructions using a globally distributed set of 80 ground-based GPS receivers from the IGS receiver network were obtained for the month of October 2012. Observations of the S4 index from the COSMIC constellation were also obtained for the same period. The COSMIC S4 estimates are provided as the RMS intensity fluctuations of the square of the L1 CA SNR at a 1 second time resolution during the occultation pass. In order to isolate F-region scintillation, the data for each pass was clipped such that the point of closest approach to the Earth for each ray path was within the height range of $200 \mathrm{~km}$ to $600 \mathrm{~km}$. The S4 estimate for the occultation event was then defined as the maximum observed within this reduced data set. Owing to the geometry of the limb ray through the ionosphere, the exact horizontal location of the scintillating structure is ambiguous to within typically $500 \mathrm{~km}$ to 800 $\mathrm{km}$. This however is the same order of magnitude as the MIDAS grid resolution and was therefore was considered sufficient for comparison with MIDAS output. 


\section{1-Oct-2012 02:00:00 UTC}

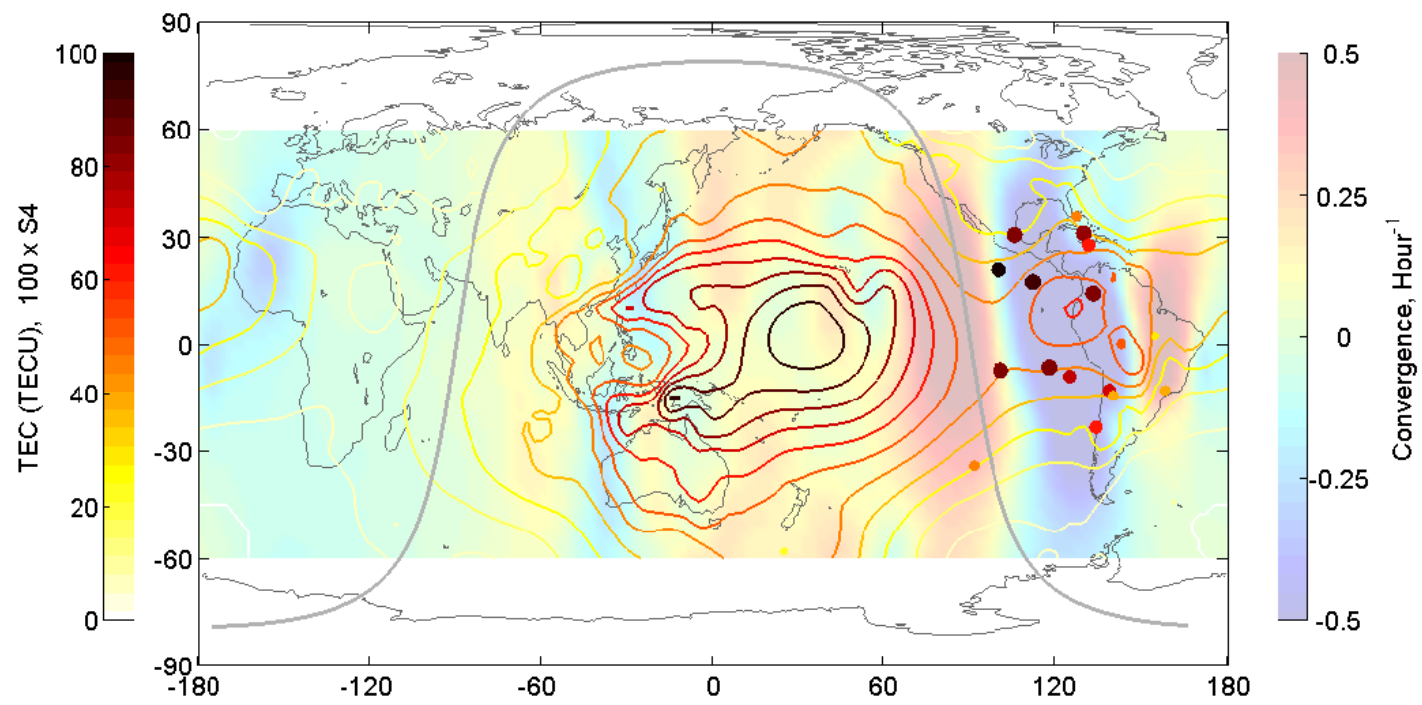

02-Oct-2012 18:40:00 UTC

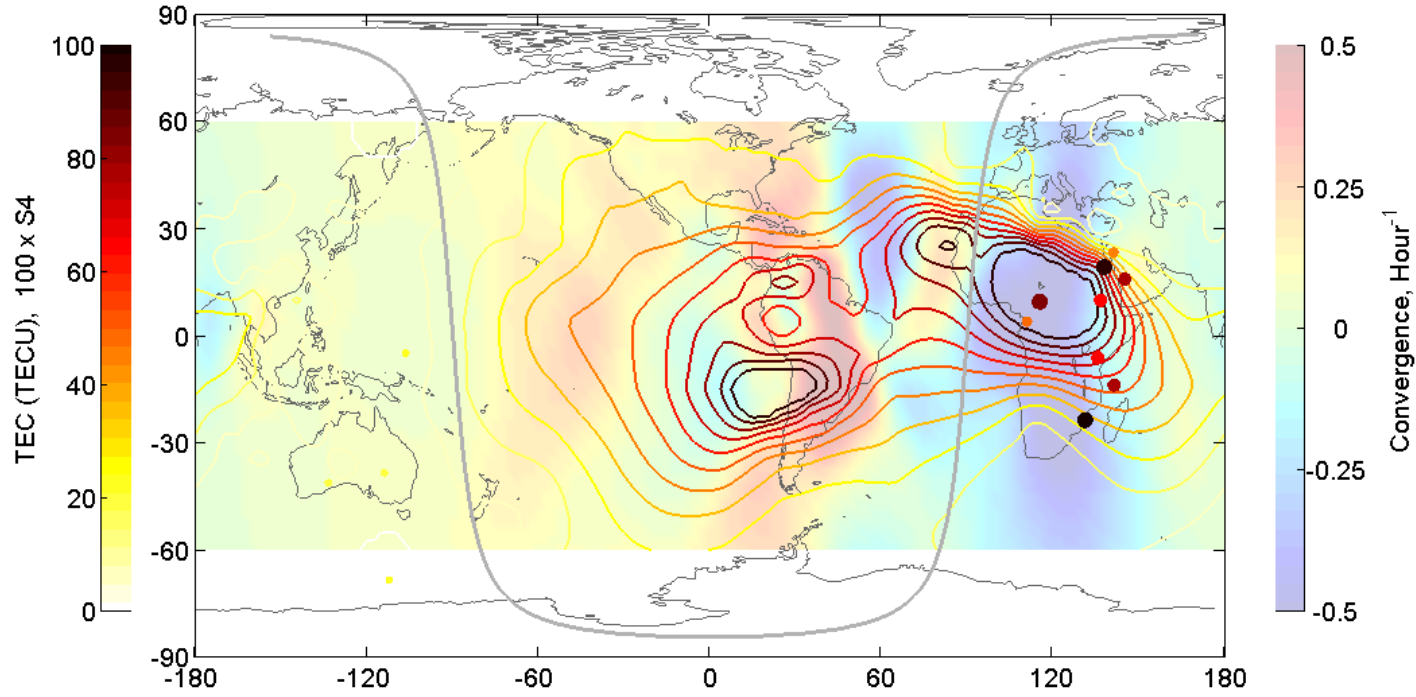

Figure 1. MIDAS reconstructions on 01/10/12 at 02:00 UTC, above, and 02/10/2012 at 18:40 UTC, below. Line contours show total electron content (left color bar). Filled contours show eastwards plasma convergence (right color bar). The thick grey line represents the solar terminator and the filled circles show maximum 44 per occultation observed by COSMIC (colored as $S 4 \times 100$ on the left color bar).

\section{Results}

Results are presented for the month of October 2012 which was chosen due to the large number of scintillation events that were observed. Figure 1 presents two examples of the output from MIDAS and the corresponding estimates of the zonal plasma convergence obtained by solution of the continuity equation. Line contours show the estimated TEC and filled contours show the estimated convergence of the eastwards velocity field. Patterns of plasma convergence and divergence can be clearly observed in the post sunset sector. Superimposed on these images are colored dots showing the maximum observed S4 per occultation pass for data within a 20 minute time window of each inversion. Multiple points imply that scintillation was occurring on multiple occultation passes with differing geometries. These plots show a coincidence of multiple scintillation events where the velocity field is strongly divergent, shown in blue.

Defining a scintillation event as the maximum S4 observed on a single occultation pass for the subset of ray paths within the F-region. A cluster of events is defined as a point for which the sum of $\mathrm{S} 4$ events within $20^{\circ}$ latitude and longitude and 20 minutes in time was greater than 3. A total of 123 such clusters occurred in the month of October 2012. Figure 2 shows the mean zonal convergence pattern centred upon the location of the clusters for the month of October 2012. The observed pattern shows that severe scintillation clusters consistently occur close to the point of minimum con- 


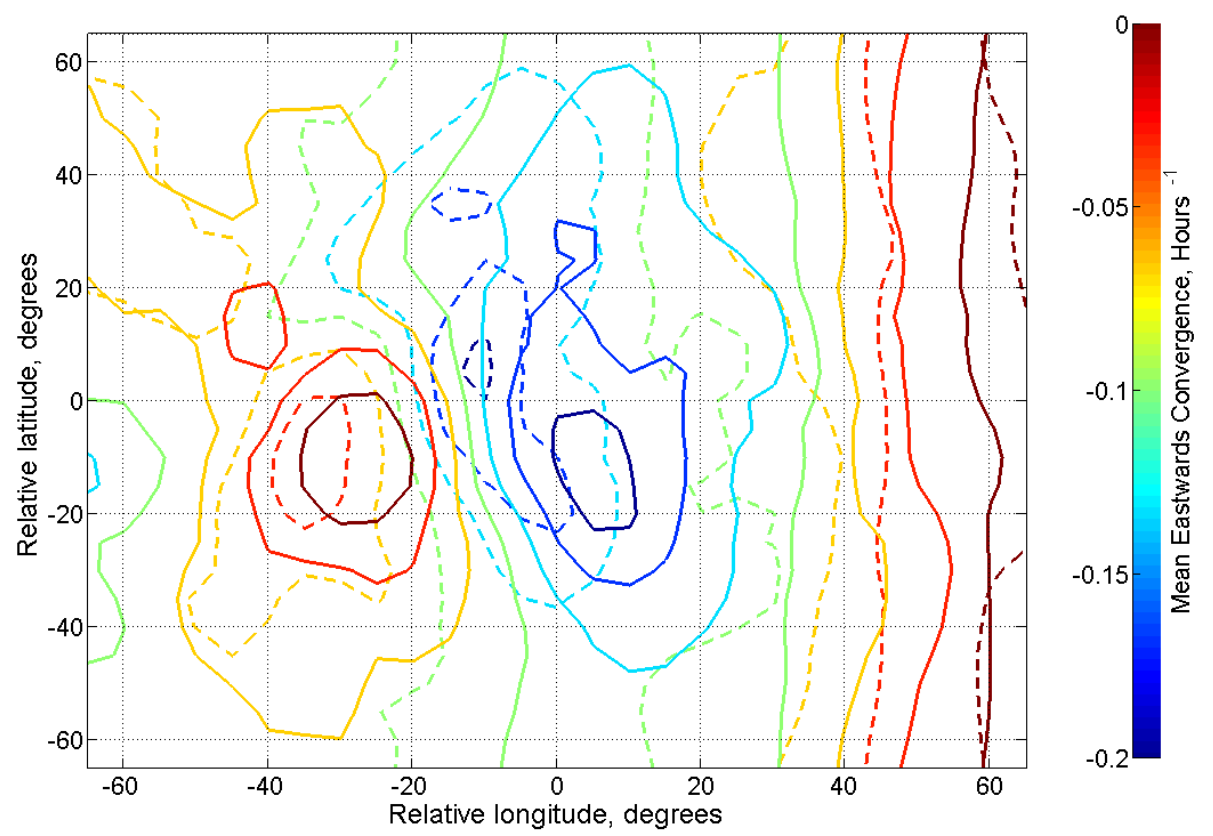

Figure 2. Mean zonal TEC convergence relative to the centre of clusters of S4 events. Solid lines represent the convergence field at time of maximum $\mathrm{S} 4$ and dotted lines 20 minutes prior.

vergence (maximum divergence). Figure 2 also shows the mean convergence field at 20 minutes prior to the time of maximum scintillation. In this case the regions of maximum convergence and divergence are shifted westwards though still clearly visible.

\section{Discussion}

Tomographic reconstructions provide a valuable resource for imaging the large scale structures in the Earth's ionosphere. The method presented uses an estimate of the divergence of the total electron content to infer the location and strength of the post-sunset drift vortex. The aim of such an approach is to provide a warning for subsequent scintillation over an extended region of space at length scales corresponding to the resolution of the MIDAS reconstructions. A significant problem in relating the occurrence of scintillation to the MIDAS results is that, at L-band frequencies, the structures that give rise to scintillation are highly localised in space and time. This problem was addressed by using $\mathrm{S} 4$ estimates from the COSMIC satellite constellation. Occulting ray paths traverse a large horizontal region of the ionosphere. The occultation geometry is therefore better suited to the current analysis by providing a spatially averaged scintillation index at the approximate resolution of the MIDAS grid. Furthermore, clusters of scintillation events observed on multiple occultation passes also imply that the irregularities exist over an extended spatial region.

The results presented in Figure 1 show two examples of the TEC and estimated TEC divergence. In both cases a post sunset enhancement in the TEC is apparent with the TEC enhancement on the 2nd October being approximately twice that of the event of the previous day. It can also be seen that the gradients in TEC are significantly different for the two events. The number of scintillation events and TEC divergence are however similar in both cases.

In order to test whether TEC divergence can consistently be used to predict the likely occurrence of scintillation the mean convergence pattern centred on all severe scintillation clusters is shown in Figure 2. Close to the centre of the plot the zonal TEC convergence is negative with a localised region of positive convergence to the east. This pattern is common across the large number of severe scintillation events observed during the month of October 2012. It is also apparent from the dotted contours that the pattern forms prior to the occurrence scintillation clusters. Consequently these patterns of convergent and divergent TEC can be used as an advanced warning of scintillation.

\section{Conclusion}

The goal of this paper is to present a method for forecasting the location of scintillation events at L-band frequencies using GPS based tomographic reconstructions of the ionosphere. The method relies on the close relationship between the post-sunset drift vortex and the pre-reversal enhancement in the eastwards electric field. The lifting of the plasma due to the eastwards electric field on the day side of the drift vortex is related to an increase in the eastwards plasma velocity above the vortex. The later causing a zonal divergence of the total electron content that can be imaged from tomographic reconstructions. Future work will aim to relate the results obtained here to in-situ observations of electric fields obtained directly by independent satellite observations. 
Acknowledgements. The MIDAS software package is used under license from the Department of Electronic and Electrical Engineering, University of Bath, Bath, BA2 7AY.

\section{References}

Anderson, D.N., B. Reinisch, C. Valladares, C. Chau and O. Veliz (2004). Forecasting the occurrence of ionospheric scintillation activity in the equatorial ionosphere on a day-to-day basis, Journal of Atmospheric and Solar-Terrestrial Physics, 66 (17), 1576-1572.

Bittencourt, J.A., V.G. Pillat, P.R. Fagundes, Y. Sahai and A.A. Pimenta (2007). LION: A dynamic computer model for the low-latitude ionosphere, Annales Geophysicae, 25, 2371-2392.

Fejer, B.G., and M.C. Kelley (1980), Ionospheric irregularities, Reviews of Geophysics, 18 (2), 401-454.

Kudeki, E., A. Akgiray, M. Milla, J.L. Chau, D.L. Hysell (2007). Equatorial spread-F initiation: Post-sunset vortex, thermospheric winds, gravity waves, Journal of Atmospheric and Solar-Terrestrial Physics, 69 (17-18), 2416-2427.

Mitchell, C.N., and P.S.J. Spencer (2003). A three-dimensional time-dependent algorithm for ionospheric imaging using GPS, Annals of Geophysics, 46 (4), 687-696.

Redmond, R.J., D. Anderson, R. Caton and T. Bullett (2010). A Forecasting Ionospheric Real-time Scintillation Tool (FIRST), Space Weather, 8 (12), S12003.

Rodrigues, F.S., G. Crowley, R.A. Heelis, A. Maute and A. Reynolds (2012). On TIE-GCM simulation of the evening equatorial plasma vortex, Journal of Geophysical Research, 117, A05307.

Straus, P., and R. Bishop (2009). Radio Occultation Measurements of Ionospheric Scintillation, Presented at the 4th COSMIC Data Users Workshop, October 2009, Boulder, CO, USA.

Uma, G., J.Y. Liu, P.S. Brahmanandam, C.H. Chen, Y. Kakinami and C.H. Liu (2009). A Global Observation of S4 Scintillation Index Observed by Means of FORMOSAT-3/COSMIC, Presented at the 4th COSMIC Data Users Workshop, October 2009, Boulder, CO, USA.

\footnotetext{
${ }^{\star}$ Corresponding author: Paul S.J. Spencer,

European Commission, Joint Research Center (JRC), IPSC, I-21027

Ispra (Varese), Italy; email: paul.spencer@jrc.ec.europa.eu.

(C) 2014 by the Istituto Nazionale di Geofisica e Vulcanologia. All

rights reserved.
} 